DOI: $10.1111 /$ cea.13230

Asthma and Rhinitis

\title{
Electronic Clinical Decision Support System for allergic rhinitis management: MASK e-CDSS
}

Anne-Lise Courbis $^{1}$ | Ruth Brigid Murray ${ }^{2}$ | Sylvie Arnavielhe ${ }^{3}$ | Davide Caimmi ${ }^{4}$ | Anna Bedbrook $^{5}$ | Michiel Van Eerd ${ }^{6}$ | Govert De Vries ${ }^{6}$ | Gerard Dray ${ }^{1}$ |

${\text { loana } \text { Agache }^{7} \text { | Mario Morais-Almeida }}^{8}$ | Claus Bachert ${ }^{9}$ | Karl Christian Bergmannn ${ }^{10}$ |

Sinthia Bosnic-Anticevich ${ }^{11}$ | Jan Brozek ${ }^{12}$ | Caterina Bucca ${ }^{13}$ | Paulo Camargos ${ }^{14}$ |

Giorgio Walter Canonica ${ }^{15}$ | Warner Carr ${ }^{16}$ | Thomas Casale ${ }^{17}$ | Joao A. Fonseca ${ }^{18}$ |

Tari Haahtela $^{19}$ | Omer Kalayci ${ }^{20}$ | Ludger Klimek $^{21}$ | Piotr Kuna ${ }^{22}$ |

Violeta Kvedariene $^{23}$ | Desiree Larenas Linnemann ${ }^{24}$ | Phil Lieberman ${ }^{25}$ |

Joaquim Mullol $^{26}$ | Robyn Ohehir ${ }^{27}$ | Nikolaos Papadopoulos ${ }^{28,29}$ | David Price ${ }^{30}$ |

Dermot Ryan $^{31}$ | Boleslaw Samolinski ${ }^{32}$ | F. Estelle Simons ${ }^{33}$ | Peter Tomazic ${ }^{34}$ |

Massimo Triggiani $^{35}$ | Arunas Valiulis $^{36}$ | Erkka Valovirta ${ }^{37,38}$ | Martin Wagenmann ${ }^{39}$ |

Magnus Wickman $^{40}$ | Arzu Yorgancioglu ${ }^{41}$ | Jean Bousquet ${ }^{5,42,43,44}$

${ }^{1}$ LGI2P, IMT Mines Ales, University of Montpellier, Ales, France

${ }^{2}$ Department of Allergic Diseases, Medscript NZ Ltd, Paraparaumu Beach, Kapiti Coast, New Zealand

${ }^{3}$ Kyomed, Montpellier, France

${ }^{4}$ Department of Respiratory Diseases, Montpellier University Hospital, Montpellier, France

${ }^{5}$ Contre les MAladies Chroniques pour un Vleillissement Actif en France European Innovation Partnership on Active and Healthy Ageing Reference Site,

Montpellier, France

${ }^{6}$ Peercode B.V., Geldermalsen, The Netherlands

${ }^{7}$ Transylvania University Brasov, Brasov, Romania

${ }^{8}$ Immunoallergy Department, CUF Descobertas Hospital, Lisbon, Portugal

${ }^{9}$ ENT Department, Upper Airways Research Laboratory, Ghent University Hospital, Ghent, Belgium

${ }^{10}$ Allergy-Centre-Charité, Department of Dermatology and Allergy, Charité - Universitätsmedizin Berlin, Berlin, Germany

${ }^{11}$ Woolcock Institute of Medical Research, University of Sydney and Sydney Local Health District, Glebe, New South Wales, Australia

${ }^{12}$ Department of Clinical Epidemiology and Biostatistics, McMaster University, Hamilton, Ontario, Canada

${ }^{13}$ Hospital City of Health and Science of Torino, University Pneumology Unit-AOU Molinette, Turin, Italy

${ }^{14}$ Department of Pediatrics, Medical School, Federal University of Minas Gerais, Belo Horizonte, Brazil

${ }^{15}$ Personalized Medicine Asthma \& Allergy Clinic, Humanitas University \& Research Hospital, Milan, Italy

${ }^{16}$ Allergy and Asthma Associates of Southern California, Mission Viejo, California, USA

${ }^{17}$ Division of Allergy and Immunology, University of South Florida Morsani College of Medicine, Tampa, Florida, USA

${ }^{18}$ CINTESIS, Faculdade de Medicina da Universidade do Porto \& MEDIDA, LDA, Porto, Portugal

${ }^{19}$ Skin and Allergy Hospital, Helsinki University Hospital, Helsinki, Finland

${ }^{20}$ Department of Pediatric Allergy, Hacettepe University School of Medicine,Ankara, Turkey

${ }^{21}$ Center for Rhinology and Allergology, Wiesbaden, Germany

${ }^{22}$ Barlicki University Hospital, Medical University of Lodz, Lodz, Poland 


\footnotetext{
${ }^{23}$ Department of Pathology, Faculty of Medicine, Clinic of Infectious, Chest Diseases, Dermatology and Allergology, Institute of Biomedical Sciences, Vilnius University, Vilnius, Lithuania

${ }^{24}$ Clínica de Alergia, Asma y Pediatría, Hospital Médica Sur, Mexico City, Mexico

${ }^{25}$ Divisions of Allergy and Immunology, Department of Internal Medicine and Pediatrics, University of Tennessee College of Medicine, Germantown, Tennessee, USA

${ }^{26}$ ENT Department, Hospital Clinic, Clinical and Experimental Respiratory Immunoallergy, IDIBAPS, CIBERES, Universitat de Barcelona, Barcelona, Spain

${ }^{27}$ Department of Allergy, Immunology and Respiratory Medicine, Alfred Hospital and Central Clinical School, Monash University, Melbourne, Victoria, Australia

${ }^{28}$ Division of Infection, Immunity \& Respiratory Medicine, University of Manchester, Manchester, UK

${ }^{29}$ Allergy Department, 2nd Pediatric Clinic, University of Athens, Athens, Greece

${ }^{30}$ Observational and Pragmatic Research Institute, Singapore, Singapore

${ }^{31}$ Usher Institute of Population Health Sciences and Informatics, University of Edinburgh Medical School, Edinburgh, UK

${ }^{32}$ Department of Prevention of Environmental Hazards and Allergology, Medical University of Warsaw, Warsaw, Poland

${ }^{33}$ Department of Pediatrics and Child Health, Department of Immunology, Faculty of Medicine, University of Manitoba, Winnipeg, Winnipeg, Canada

${ }^{34}$ Department of ENT, Medical University of Graz, Graz, Austria

${ }^{35}$ Division of Allergy and Clinical Immunology, University of Salerno, Salerno, Italy

${ }^{36}$ Department of Public Health, Institute of Clinical Medicine, Clinic of Children's Diseases, Institute of Health Sciences, Faculty of Medicine, Vilnius University, Vilnius, Lithuania

${ }^{37}$ Department of Lung Diseases and Clinical Allergology, University of Turku, Turku, Finland

${ }^{38}$ Allergy Clinic, Terveystalo, Turku, Finland

${ }^{39}$ Department of Otorhinolaryngology, HNO-Klinik, Universitätsklinikum Düsseldorf, Düsseldorf, Germany

${ }^{40}$ Centre for Clinical Research Sörmland, Uppsala University, Eskilstuna, Sweden

${ }^{41}$ Department of Pulmonology, Faculty of Medicine, Celal Bayar University, Manisa, Turkey

${ }^{42}$ INSERM U 1168, VIMA: Ageing and Chronic Diseases Epidemiological and Public Health Approaches, Villejuif, France

${ }^{43}$ UMR-S 1168, Université Versailles St-Quentin-en-Yvelines, Montigny le Bretonneux, France

${ }^{44} \mathrm{CHU}$ Montpellier, Montpellier, France
}

\section{Correspondence:}

Jean Bousquet, CHU Montpellier, 371

Avenue du Doyen Gaston Giraud, 34295

Montpellier Cédex 5, France.

Email: jean.bousquet@orange.fr.

\section{Summary}

Background: Allergic rhinitis (AR) management has changed in recent years following the switch from the concept of disease severity to the concept of disease control, publication of the AR clinical decision support system (CDSS) and development of mobile health (m-health) tools for patients (eg Allergy Diary). The Allergy Diary Companion app for healthcare providers is currently being developed and will be launched in 2018. It incorporates the AR CDSS to provide evidencebased treatment recommendations, linking all key stakeholders in $A R$ management.

Objective: To produce an electronic version of the AR CDSS (e-CDSS) for incorporation into the Allergy Diary Companion, to describe the app interfaces used to collect information necessary to inform the e-CDSS and to summarize some key features of the Allergy Diary Companion.

Methods: The steps involved in producing the e-CDSS and incorporating it into the Allergy Diary Companion were (a) generation of treatment management scenarios; (b) expert consensus on treatment recommendations; (c) generation of electronic decisional algorithms to describe all AR CDSS scenarios; (d) digitization of these algorithms to form the e-CDSS; and (e) embedding the e-CDSS into the app to permit easy user e-CDSS interfacing.

Results: Key experts in the AR field agreed on the AR CDSS approach to AR management and on specific treatment recommendations provided by Allergy Diary Companion. Based on this consensus, decision processes were developed and 
programmed into the Allergy Diary Companion using Titanium Appcelerator (Java-

Script) for IOS tablets. To our knowledge, this is the first time the development of any $m$-health tool has been described in this transparent and detailed way, providing confidence, not only in the app, but also in the provided management recommendations.

Conclusion: The Allergy Diary Companion for providers provides guideline and expert-endorsed AR management recommendations. [MASK paper No 32].

\section{1 | INTRODUCTION}

The aim of allergic rhinitis (AR) management is to achieve control of the disease and its symptoms ${ }^{1,2}$ and is a dynamic process. Control can fluctuate over time influenced by many factors, including $A R$ phenotype (ie intermittent or persistent $A R$ ), environmental exposure and current treatment. Therefore, the tool used to measure disease control must be simple, suitable for everyday use and sensitive to change. A simple visual analogue scale (VAS) has been recommended as the language for assessing AR control. ${ }^{2-4}$ It has recently been converted to electronic format and included in a free mobile app for patients-Allergy Diary —as part of MASK (Mobile Airways Sentinel network). ${ }^{5-8}$ The Allergy Diary aims to empower patients to self-manage their AR., 5 The use of self-management and information and communication technology may hold the key to chronic disease management and provides useful objective data for physicians.

The VAS has also been incorporated into an AR clinical decision support system (CDSS), a new type of disease management tool. ${ }^{2} \mathrm{~A}$ CDSS is a health information technology system, based on the best evidence and algorithms, designed to provide physicians, and other healthcare providers, with clinical decision support (ie assistance with clinical decision-making tasks). It is an important tool for precision medicine., ${ }^{9,10}$ Within the AR CDSS, the VAS is used to assess AR control and to assist in making management decisions, assisting patients and healthcare providers to jointly determine AR treatment and its step-up or step-down strategy depending on the status of AR control. $^{2}$

The next step is to integrate the AR CDSS into an app for providers called Allergy Diary Companion, by producing an electronic version of it (e-CDSS). The Allergy Diary Companion app is designed for use during a patient-physician consultation. The e-CDSS will effectively be the "brain" of the app, providing guideline-directed AR management recommendations in response to 4 inputs: VAS score, Allergic Rhinitis \& its Impact on Asthma (ARIA)-defined AR phenotype, allergen exposure and AR treatment history. This article describes the production of the e-CDSS from the paper version ${ }^{2}$ for incorporation into the Allergy Diary Companion. Expert consensus on AR CDSS recommendations, as well as the production of decision processes to describe this consensus and programme the app, is also described. Finally, description of the various interfaces used by the app to collect information necessary to inform the e-CDSS and key features of Allergy Diary Companion are summarized.

\section{2 | METHODS}

Figure 1 outlines the six steps involved in the transformation of the paper AR CDSS (Figure S1A and B) ${ }^{2}$ to the e-CDSS

\section{1 | Step 1: Major scenarios on AR management approach}

Thirteen major scenarios were identified from the AR CDSS (Table S1). These scenarios incorporated VAS score cut-offs, ARIA-defined AR phenotype (ie intermittent or persistent $A R$ ) and AR treatment, and provided an AR management approach (ie when to initiate treatment, continue, step-up and step-down treatment). T0 represents no treatment. For the purpose of these scenarios, AR treatments were coded as follows:

- T1: Non-sedating $\mathrm{H}_{1}$-antihistamines (oral, intranasal and intraocular), leukotriene receptor antagonists (LTRA) and cromones (intranasal or intraocular)

- T2: Intranasal corticosteroid (INCS)

- T3: INCS + azelastine (AZE)

- T4: Oral corticosteroids (as add on to local treatment)

- T5: Consider referral to an allergist and allergen immunotherapy (AIT)

The AR CDSS is an algorithm for the acute symptomatic treatment of AR. Recommendations on the benefits and use of AIT may be found elsewhere. ${ }^{11}$

\subsection{Step 2: Specific AR treatment recommendations}

From these 13 major scenarios, 34 detailed scenarios were developed. $^{2}$ Inputs considered included disease phenotype (ie intermittent/persistent), allergen exposure, VAS score and current treatment and specific AR management recommendations were provided (Tables S2-S5). 

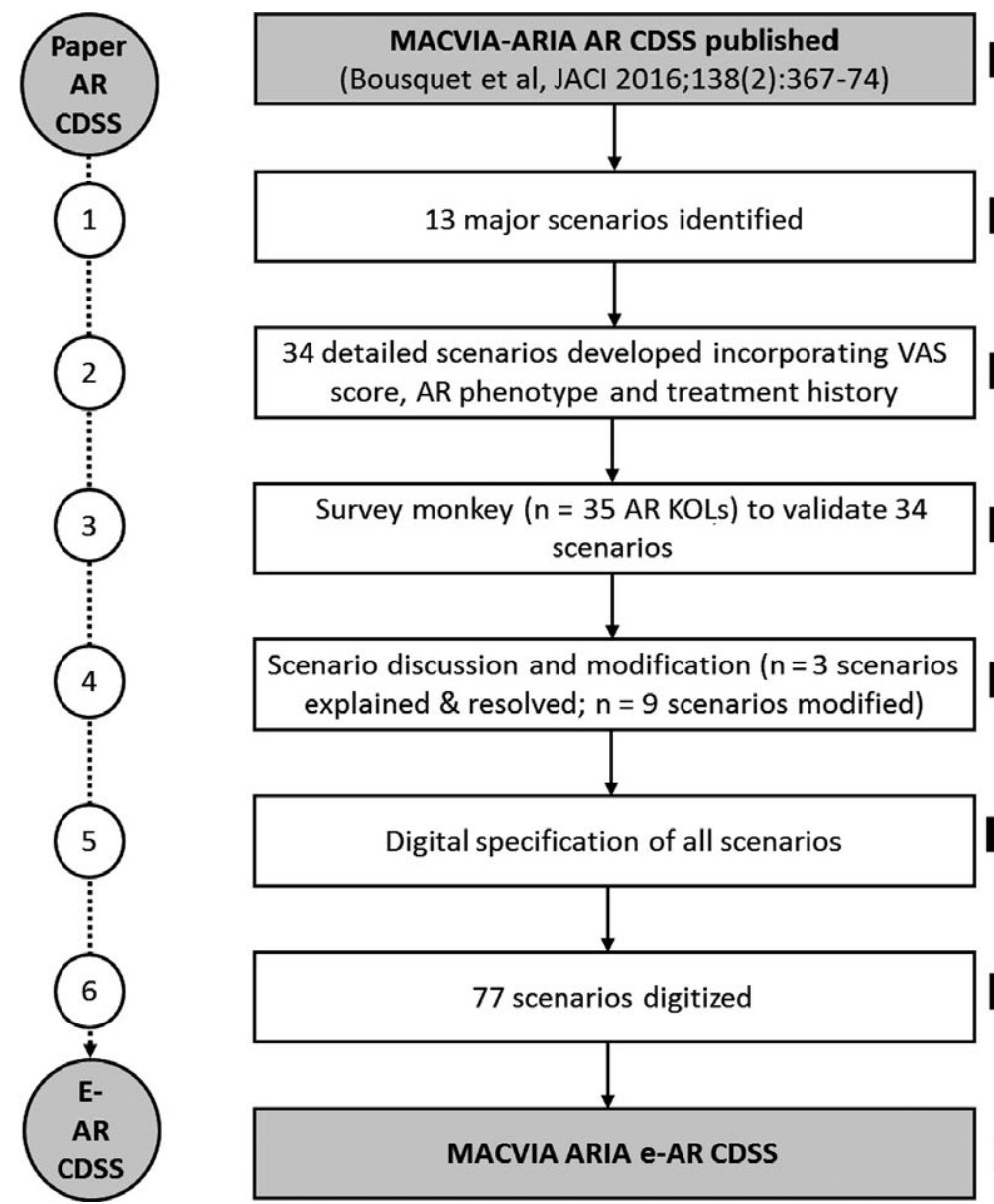

$\longrightarrow$ Paper AR CDSS

When to initiate, continue, step-up
or step-down AR treatment

Specific AR treatment

recommendations provided

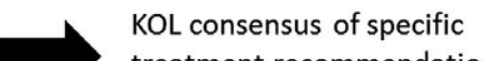

treatment recommendations

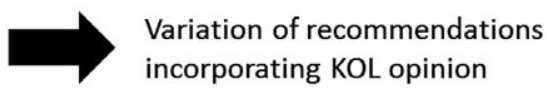

incorporating KOL opinion

Production of electronic algorithm

Accounting for all possible patient and physician
inputs into Allergy Diary Companion app

FIGURE 1 Steps for the development of the ARIA e-CDSS. MACVIA, Contre les Maladies Chroniques pour un Vieillissement actif; ARIA, Allergic Rhinitis and its Impact on Asthma; AR, allergic rhinitis; CDSS, clinical decision support system; VAS, visual analogue scale; KOL, key opinion leader

\section{3 | Step 3: Survey of experts}

These 34 detailed scenarios were incorporated into a survey that was delivered by an online service, Survey Monkey (www.survey monkey.com), and sent to 70 experts on AR and ARIA members from 23 countries, in order to achieve consensus and improve robustness of the AR CDSS algorithm. Experts were selected for their expertise (ie general practice, allergy, ENT, respiratory medicine, pharmacy and public health) with a global representation. We aimed at a 50\% response rate. The survey was divided into 4 parts: (a) general approach to AR treatment (ie when to initiate, continue, step-up or step-down treatment; $\mathrm{n}=8$ questions; Table S2); (b) treatment step-up (ie what treatment to step-up to considering current treatment; $\mathrm{n}=11$ questions; Table S3); (c) treatment stepdown (ie what treatment to step-down to considering current treatment; $\mathrm{n}=12$ questions; Table S4); and (d) treatment initiation (ie what treatment to start with, considering VAS score and AR phenotype; $n=3$ questions; Table S5). Experts indicated their level of agreement for each of the 34 presented scenarios on a VAS ranging from $0 \mathrm{~mm}$ (strongly disagree) to $100 \mathrm{~mm}$ (strongly agree). A response was returned by 35 experts (response rate: $50 \%$ ) from the USA and Canada $(n=5)$, Europe $(n=26)$, South America $(n=2)$ and Australia $(n=2)$.

Individual scores for each of the 34 scenarios were tabulated, averaged and categorized in the classical way: survey VAS score 0-25 mm (poor agreement); 25-49 mm (fair agreement); 50-74 mm (good agreement); and 75-100 mm (excellent agreement) (Figure S2A). Excellent expert consensus was achieved for all step-up scenarios except scenarios 11 (survey VAS score: $64 \mathrm{~mm}$ ), 13 (survey VAS score: $64 \mathrm{~mm}$ ) and 14 (survey VAS score: $64 \mathrm{~mm}$ ) which all dealt with step-up from T3 (Table S3; Figure S2A). Excellent expert consensus was also achieved for all step-down scenarios except scenarios 20 (survey VAS score: $74 \mathrm{~mm}$ ) and 21 (survey VAS score: $66 \mathrm{~mm}$ ) which dealt with step-down from T3 or T2, respectively (Table S4; Figure S2A). The reasons for these lower consensus scores and resolution of these issues are provided in the online Supporting information.

\section{4 | Step 4: Scenario modification and summary of findings}

An email was sent to all experts who provided a survey VAS score $<50 \mathrm{~mm}$ for any scenario to (a) understand the reason for 
disagreement and (b) achieve consensus on a resolution. Experts were permitted to change their original survey VAS scores based on the results of this discussion process (these changes are shown in red font in Figure S2B). Expert agreement increased to a VAS score $>75$ for all scenarios that were considered a second time. Details of expert comments on the scenarios, and the response and resolution are provided in the online Supporting information.

\subsection{Step 5: Digital specifications of AR CDSS (all scenarios and modifications)}

The next step was to digitally specify the agreed scenarios to generate a series of digital rules used to programme the Allergy Diary Companion (Ecole des Mines, Ales). The first task was to define a set of representative scenarios with experts of the domain, taking into account all possible parameters (eg VAS, ongoing treatment and type of patient). Then, scenarios were translated into a graphical representation, providing an algorithm, which allowed the recommendations to be automatically defined (Figure 2 A-D). The algorithm was validated in several ways: (a) review with experts, (b) review of recommendations and (c) transformation of the algorithm into a state machine (ie a model representing the state cycle of a patient.) The process enabled verification that the patient state may evolve, depending on adequate value of VAS and according to all possible treatment transitions (ie from "no treatment" to "under treatment", staying "under treatment" and come back to "no treatment"). These verifications ensured that all possible situations were taken into account in a systematic way.

\section{6 | Step 6: Programming}

Finally, a total of 77 scenarios (all possible scenarios) were programmed into the Allergy Diary Companion. The app was programmed and designed by Peercode, Netherlands, using the digital specifications above (including expert treatment recommendations), ensuring consideration of each possible input into the app. It was developed in Titanium Appcelerator (JavaScript) for tablets on the IOS platform and is easily translated and modified. Usability of the app and reliability of the treatment recommendations it provides has been checked by Peercode and further validation is currently ongoing prior to launch. The app requires no Internet function after download.

\section{RESULTS}

\subsection{Summary of survey results}

Mean scores for each question were calculated to show the level of consensus for each of the 34 AR CDSS scenarios (Table 1 and Figure S3A-D). Experts endorsed the AR CDSS approach, with good agreement achieved on step-up and step-down treatment recommendations. In summary:

- AR treatment should be stepped-up for treated AR patients with a VAS score $\geq 5 / 10 \mathrm{~cm}$.
- For patients with VAS score $\geq 2$ to $<5 / 10 \mathrm{~cm}$, treatment should be continued for patients with intermittent AR (IAR) and continued or stepped-up for those with persistent AR (PER).

- Treatment should be stepped-down for patients with a VAS score $<2 / 10 \mathrm{~cm}$.

- When step-up treatment is recommended, patients on T1 should be stepped up to T2 OR T3.

- When step-up treatment is recommended, patients on T2 should be stepped up to T3.

- Short course oral corticosteroids (ie T4) may be added here if necessary.

- Patients should be referred (eg to ENT or allergy specialists) if VAS score remains $\geq 5 / 10 \mathrm{~cm}$ or if there is a need for oral corticosteroids.

- When step-up treatment is recommended, patients on multiple therapies should be stepped up to T3 and T4 added on (short course to minimize side-effects and only if necessary).

- Stepping-down treatment was essentially the same in reverse, with the proviso that patients with nasal congestion should be stepped-down to an INCS-containing regimen in preference to T1 (albeit many patients will self-medicate and stop treatment). ${ }^{* *}$

- Treatment step-up and step-down strategies remain the same irrespective of AIT status.*

*: Does not consider the indication of AIT.

**: Modified after expert consensus.

\subsection{Digital specification of the e-CDSS modules}

To make a treatment recommendation, the CDSS within the Allergy Diary Companion must be "fed" with information. We have described this information as modules (Table 2) with each of modules 1,2 and 3 providing the input necessary for module 4 (ie e-CDSS).

\subsection{1 | Module 1 (VAS score)}

During physician consultation or pharmacist visit, patients generate a VAS score using their finger and the touchscreen functionality of an iPad, allowing selection of VAS score from 0 to $10 \mathrm{~cm}$ (inclusive). For the purpose of the e-CDSS, VAS scores are categorized as $\geq 5$ / $10 \mathrm{~cm}$ (uncontrolled AR), $\geq 2$ to $<5 / 10 \mathrm{~cm}$ (partly controlled AR) and $<2 / 10 \mathrm{~cm}$ (well-controlled AR) (Table 2), the same as those cut-offs used by Allergy Diary. These cut-offs were selected based on clinical studies in both asthma and rhinitis. ${ }^{12-17}$

\subsubsection{Module 2 (treatment classification system)}

The purpose of this module is to define the class(es) of current AR medications used by patients. A simple alphanumeric system was used for the e-CDSS, with treatments classified from TO (no treatment) up to T5 (consider referral and AIT) (Table 2). 
(A)

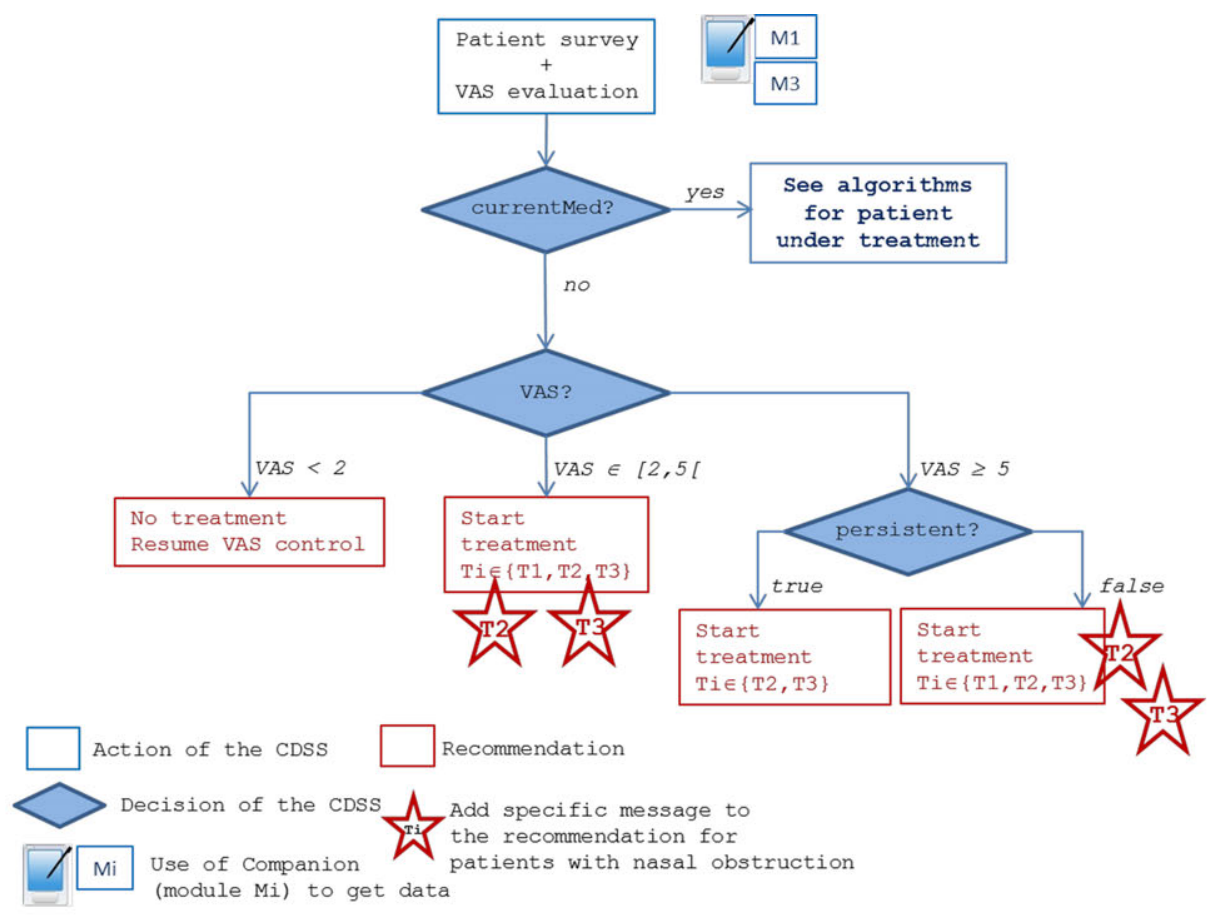

(B)

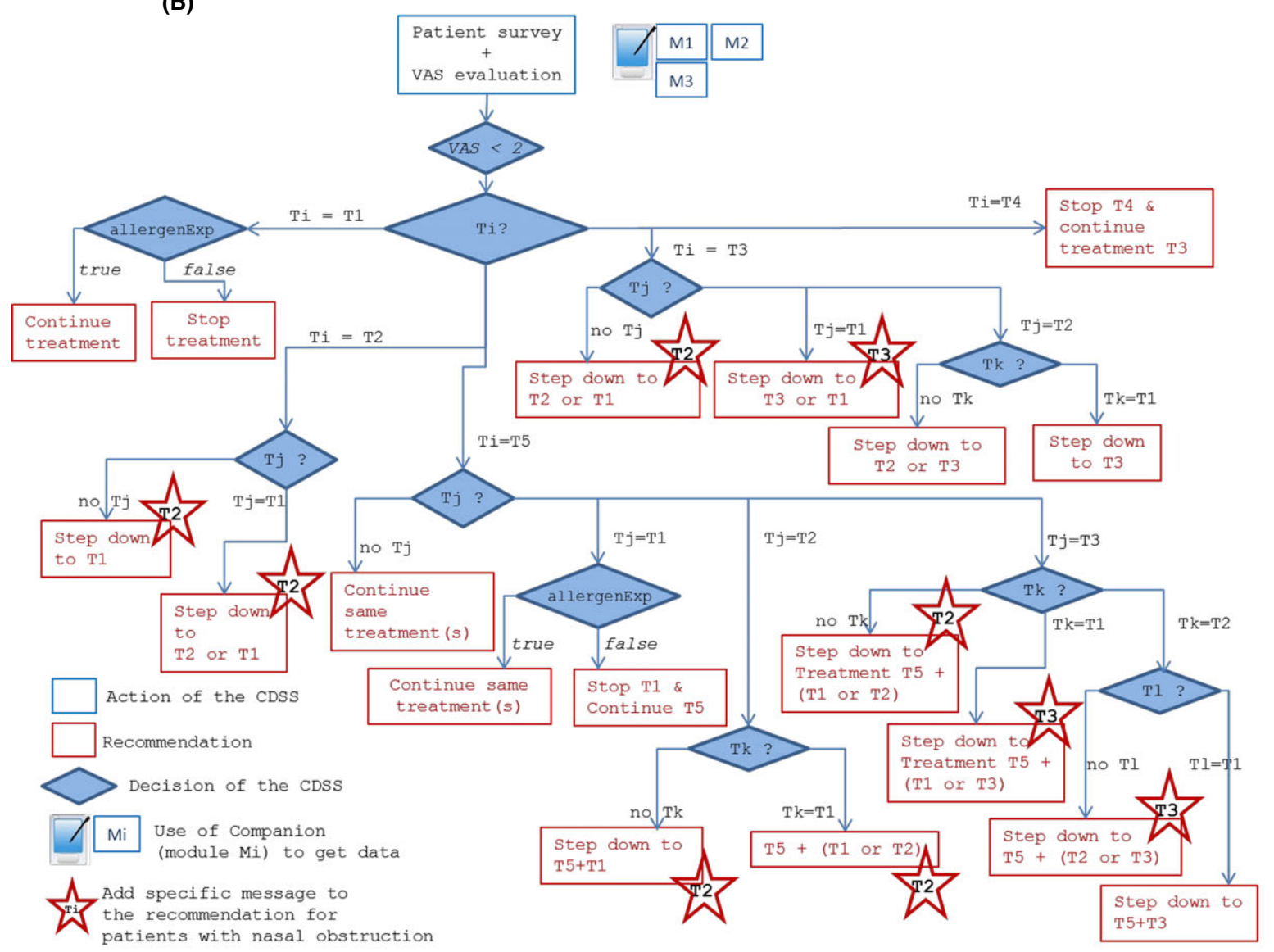

FIGURE 2 Decision processes underlying treatment recommendations for (A) patients not currently on any AR medication, (B) patients with well-controlled AR (ie VAS score $<2 / 10 \mathrm{~cm}$ ), (C) patients with partly controlled AR (ie VAS score $\geq 2$ to $<5 / 10 \mathrm{~cm}$ ) and (D) patients with uncontrolled AR (ie VAS score $\geq 5 / 10 \mathrm{~cm}$ ). AR, allergic rhinitis; VAS, visual analogue scale; M1, module 1; M2, module 2; M3, module 3; Ti, class of current treatment (in case of polypharmacy, $\mathrm{Ti}=$ maximum class). $\mathrm{Tj}, \mathrm{Tk}$ and $\mathrm{TI}$, medications added to $\mathrm{Ti}$, order of class $\mathrm{I}<\mathrm{k}<\mathrm{j}<\mathrm{i}$. $\mathrm{T} 1$, antihistamine (oral, intranasal and eye drops), leukotriene receptor antagonist, cromone (intranasal and eye drops); T2, intranasal corticosteroid (INCS); T3, INCS + Azelastine; T4 add short course of oral corticosteroids; T5, consider referral and allergen-specific immunotherapy 
(C)

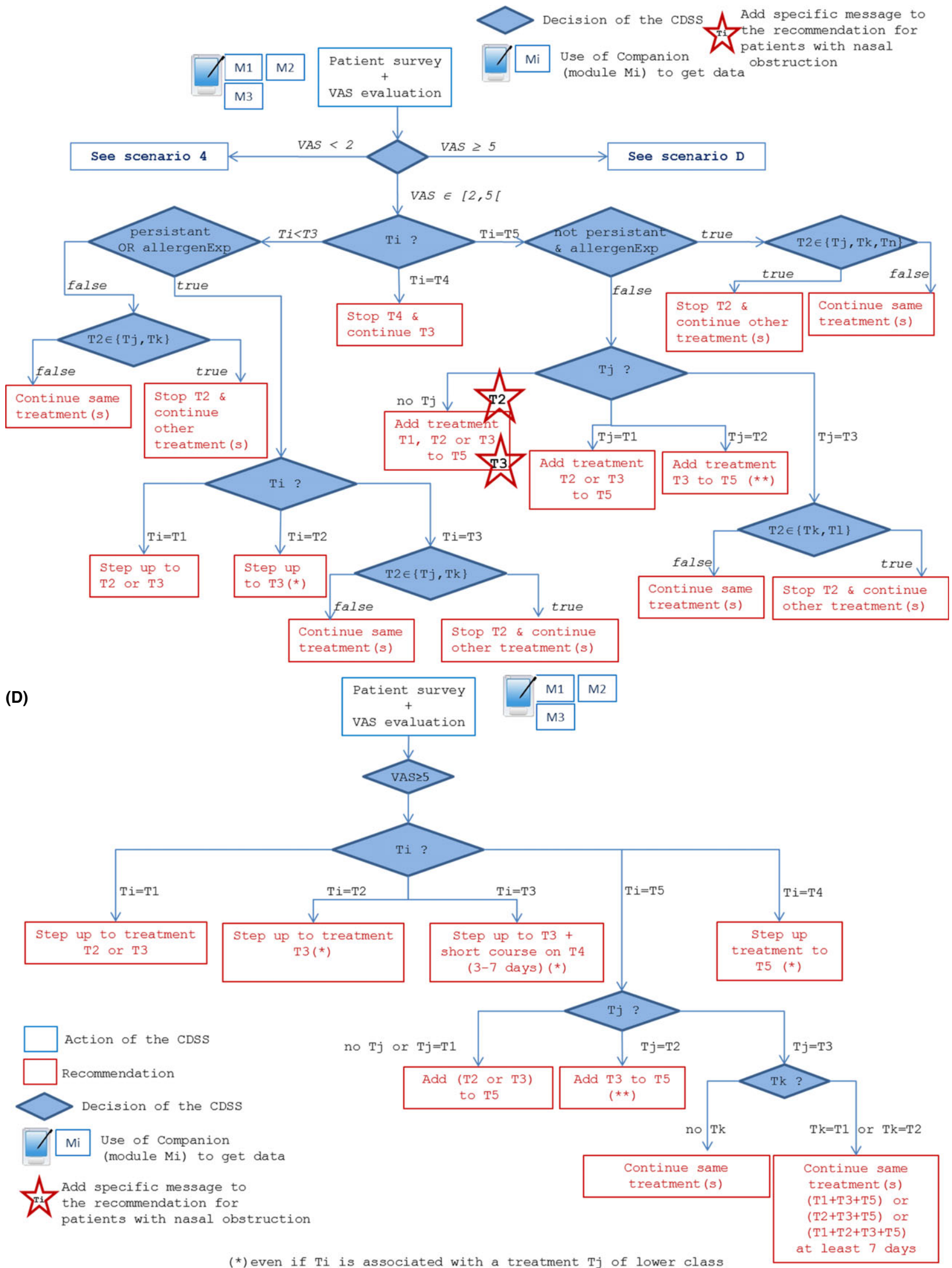

(*) even if $\mathrm{Ti}$ is associated with a treatment $\mathrm{Tj}$ of lower class $\left.{ }^{* *}\right)$ even if $\mathrm{Tj}$ is associated with a treatment $\mathrm{Tk}$ of lower class

FIGURE 2 Continued 
TABLE 1 KOL consensus opinion and level of agreement for each scenario of the AR CDSS

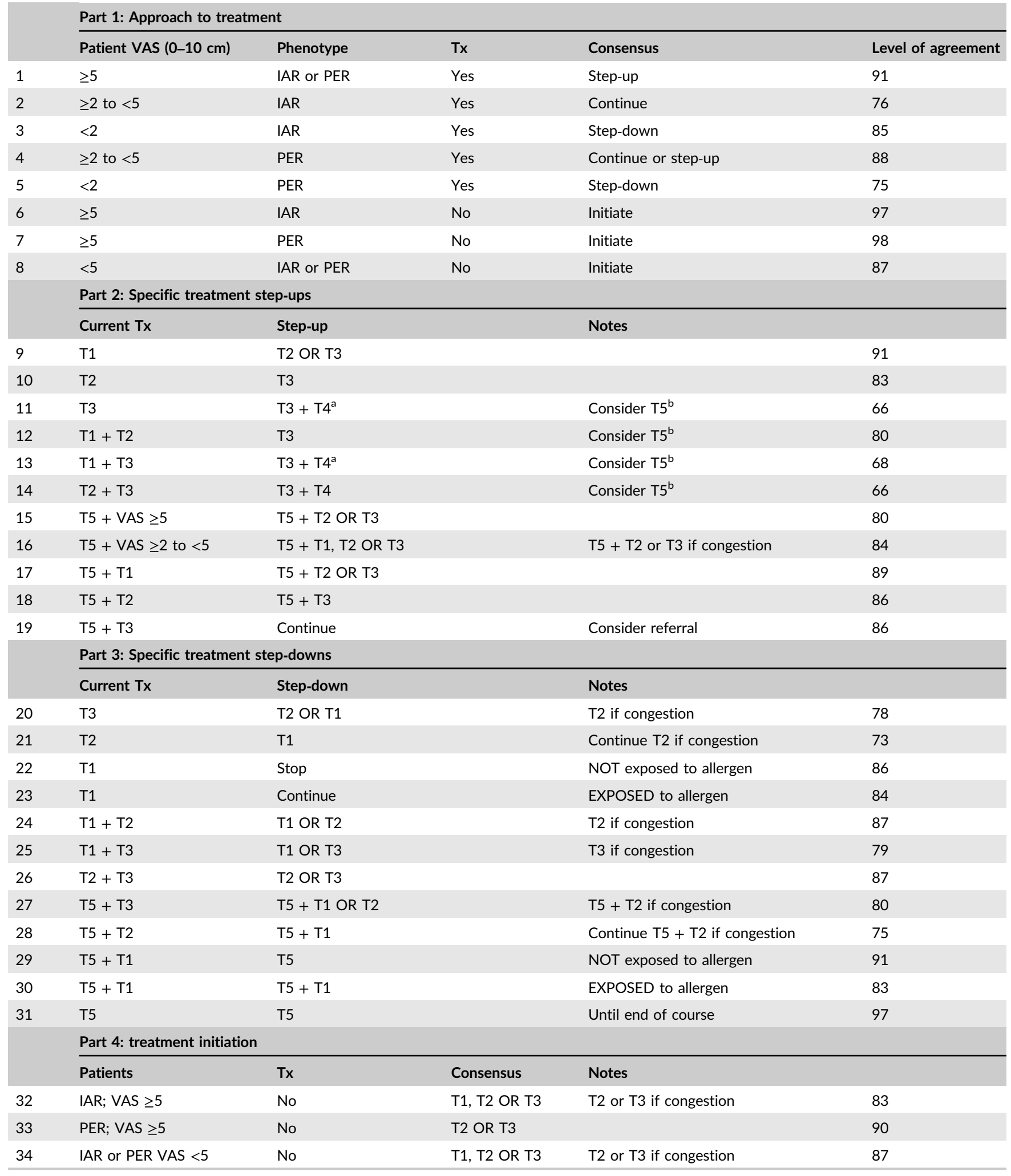

VAS: visual analogue scale; Tx: treatment; IAR: intermittent allergic rhinitis; PER: persistent allergic rhinitis; T1: antihistamine (oral, intranasal and eye drops), leukotriene receptor antagonist or cromones (intranasal and eye drops); T2: intranasal corticosteroids (INCS); T3: INCS + azelastine; T4: oral corticosteroid; T5: consider referral and allergen-specific immunotherapy; Level of agreement: VAS 275 : excellent; VAS 50-74: good.

${ }^{\text {a }}$ Short course (3-7 days)

bIf VAS score remains $\geq 5 / 10$. 
TABLE 2 Modules of e-CDSS

\begin{tabular}{|c|c|c|c|c|}
\hline & Info & Description & e-CDSS decision or input & e-CDSS variable \\
\hline M1 & VAS $(0-10 \mathrm{~cm})$ & $\begin{array}{l}<2: \text { well controlled } \\
\geq 2 \text { to }<5: \text { partly controlled } \\
\geq 5: \text { uncontrolled }\end{array}$ & $\begin{array}{c}\quad<2 \\
\geq 2 \text { to }<5 \\
\quad \geq 5\end{array}$ & {$[1 \ldots 5]$} \\
\hline \multirow[t]{5}{*}{ M2 } & \multirow{5}{*}{ Treatment classification } & None & TO & \multirow{5}{*}{$\mathrm{Ti}, \mathrm{Tj}, \mathrm{Tk}, \mathrm{TI}$} \\
\hline & & AH/LTRA/cromone & T1 & \\
\hline & & INCS & $\mathrm{T} 2$ & \\
\hline & & OC & $\mathrm{T} 4$ & \\
\hline & & Consider referral and AIT & T5 & \\
\hline \multirow[t]{5}{*}{ M3 } & \multirow[t]{5}{*}{ Patient characteristics } & Q1: Do you currently take AR medication? & Current med & \multirow[t]{2}{*}{ True/false } \\
\hline & & Q2a: Select medication(s) & Scrolled list & \\
\hline & & Q3b: How many days/week do they last (1-7)? & \multirow[t]{2}{*}{ Week $\geq 4$ \& day $\geq 4$} & \multirow{2}{*}{$\begin{array}{l}\{\text { True } / \text { false }\} \\
\text { True }=\text { PER } \\
\text { False }=I A R\end{array}$} \\
\hline & & Q4b: How many consecutive weeks do they last (1-5, or more)? & & \\
\hline & & Q5: Are you currently exposed to allergen? & \multirow[t]{2}{*}{ Yes or don't know } & $\{$ True/false $\}$ \\
\hline M4 & CDSS & Data from $M 1, M 2$ and $M 3$ & & $\begin{array}{l}\text { True }=y e s \\
\text { False }=\text { no }\end{array}$ \\
\hline
\end{tabular}

CDSS, clinical decision support system; VAS, visual analogue scale; AH, antihistamine (oral, intranasal and eye drops); LTRA, leukotriene receptor antagonist; INCS, intranasal corticosteroid; AZE, azelastine; OC, oral corticosteroid; AIT, allergen-specific immunotherapy; AR, allergic rhinitis; Tx, treatment; PER, persistent AR; IAR, intermittent AR; Ti, current treatment (if multiples $\mathrm{Ti}$ = highest medication class); $\mathrm{Tj}, \mathrm{k}, \mathrm{l}$, add on treatments to $\mathrm{Ti}$.

\subsection{3 | Module 3 (patient information)}

Patients input this information into the Allergy Diary Companion during physician consultation. The information gathered (or computed) includes:

- Specific AR medication currently used

- AR phenotype (ie IAR or PER)

O AR phenotype is classified according to duration of medication use (Questions 3a and 4a, Table 2) or duration of symptoms for untreated patients (Questions $3 \mathrm{~b}$ and $4 \mathrm{~b}$, Table 2)

- Allergen exposure status

O Information on what constitutes an allergen is provided for patients

AR medication(s) are selected from a scrolled list of all over the counter or prescribed medications available in each country (Question 2a, Table 2). Multiple medication selections are permitted. For each medication class selected, a corresponding treatment code is assigned by the system. Exposure to allergen is assessed using a simple true or false algorithm (Table 2). Figure S4 shows the organogram of the sequence of questions that patients answer in Module 3. At any step, the patient can go back to a previous question to correct his/her answer, or cancel the survey answer. No personal information is collected.

\subsection{4 | Module 4 (e-CDSS specifications)}

The e-CDSS uses data obtained from the other modules to deliver a recommendation corresponding to the most appropriate treatment. Table 2 summarizes how this information is gathered, the input used by the e-CDSS to make a decision and the variables used (online Supporting information). Information from each of these modules is used to produce treatment recommendations. The decision processes underlying treatment recommendations are shown in Figure 2 (A-D). Four decision processes were developed to cover 4 scenarios: (a) the patient is not currently on any AR medication (Figure 2A), (b) the patient has well-controlled AR (ie VAS score $<2 \mathrm{~cm}$; Figure $2 B$ ), (c) the patient has partly controlled $A R$ (ie VAS score $\geq 2$ to $<5 \mathrm{~cm}$; Figure $2 \mathrm{C}$ ) and (d) the patient has uncontrolled AR (ie VAS score $\geq$ $5 \mathrm{~cm}$; Figure 2D).

\section{3 | Interfaces specifications}

\subsection{1 | Collecting information}

Allergy Diary Companion screen interfaces for collecting VAS score for the e-CDSS is shown in Figure 3A. The VAS question is "overall 
how much are your allergic symptoms bothering you today?" and is identical to that used in the Allergy Diary for patients, ensuring that both physicians and patients are speaking the same language of AR control. This allows for interconnectivity between applications, consistency of AR control assessment and computation. The Allergy Diary Companion screen Interfaces for collecting information on AR medication use, AR phenotype and allergen exposure (Module 3) are shown in Figure S5.

\subsubsection{Summarizing information}

The following information is summarized by Allergy Diary Companion for providers (Figure 3B):

- AR disease control status message: "Your patient has [well-, partlyor un-] controlled allergic rhinitis."

- Current VAS score of the patient: this is expressed as a positive integer to one decimal place. AR control is categorized according to this VAS score, and colour coded in the same way as for the Allergy Diary -well-controlled AR: green (VAS score $<2 \mathrm{~cm}$ ); partly controlled AR: yellow (VAS score $\geq 2$ to $<5 \mathrm{~cm}$ ); and uncontrolled AR: orange: (VAS score $\geq 5 \mathrm{~cm}$ ) (Figure $3 \mathrm{~B}$ ).

- AR phenotype: This is categorized as either "intermittent" or "persistent" depending on the duration of treatment (or symptoms).

- Allergen exposure: summarized as either "yes" or "no"

- Treatment: List of currently taken AR treatments.

\subsection{3 | Treatment recommendation}

A treatment recommendation is then provided based on this information, and using the rules as outlined in the e-CDSS (Figures 2AD) and summarized in Table 1. Treatment recommendations are in the format: Recommendation—approach—specific treatment recommendation. For the example shown in Figure $3 \mathrm{~B}$, the recommendation is to step-up treatment, as the patient has a VAS score $\geq 5$ / $10 \mathrm{~cm}$, has IAR (with allergen exposure) and is currently treated (with AIT and FP_ie T5 + T2) —see Table 1 scenario 1. The recommended treatment option is to continue the AIT course and step-up symptomatic treatment to INCS + AZE (ie step-up from $\mathrm{T} 5+\mathrm{T} 2=\mathrm{T} 5+\mathrm{T} 3$ ) —see Table 1 scenario 18. By clicking on "INCS + AZE," the treatment listed is "Dymista", currently the only medication in this class.

\section{4 | DISCUSSION}

In this article, we describe a 21st century approach to AR management. $\mathrm{m}$-Health refers to the use of mobile devices to collect, collate and assess patient level health data. In this article, we focus on CDSS and $m$-health in AR and describe the process of transforming the recently published $A R C D S S^{2}$ into an e-CDSS, and how this
e-CDSS was embedded into an $\mathrm{m}$-health tool for providers called the Allergy Diary Companion.

Incorporation of the e-CDSS into the Allergy Diary Companion was achieved in a systematic and collaborative way, by (a) generating treatment management scenarios, (b) obtaining expert consensus on specific AR CDSS-informed treatment recommendations, allowing for variations in line with expert opinion; (c) generating electronic algorithms to describe all scenarios within the AR CDSS; (d) digitizing these algorithms to form the e-CDSS; and finally (e) embedding the e-CDSS into the Allergy Diary Companion app to permit easy user interfacing. In this way, the Allergy Diary Companion serves as a knowledge translation intervention for providers, a dynamic and iterative process that induces the synthesis, dissemination, exchange and ethically sound application of knowledge to improve health, ${ }^{18}$ encouraging change in behaviour in keeping with scientific evidence. Key experts in the field of AR from all over the world agreed on the AR CDSS approach to AR management and on specific treatment recommendations provided by Allergy Diary Companion and informed by VAS score, disease phenotype, allergen exposure and treatment history. To our knowledge, this is the first time the development of any $\mathrm{m}$-health tool has been described in this transparent and detailed way, providing confidence, not only in the app, but also in the treatment recommendations it provides.

The Allergy Diary Companion is essentially a decision aid for providers. Decision aids increase knowledge, reduce decisional conflict and have a positive effect on patient-practitioner communication 19,20 and may be particularly effective when incorporated into an ehealth tool. For example, when providers use handheld computers to access clinical information, their knowledge improves significantly more than peers who use paper resources. Physician adherence to guidelines is poor ${ }^{21}$ and it is hoped that when guideline recommendations are presented electronically, providers may make safer prescribing decisions and adhere more closely to these recommendations vs peers using paper resources. ${ }^{22}$ Unfortunately, at the physician level CDSSs are rarely used, and the advice is not followed, ${ }^{23}$ even though use of computerized CDSSs have been shown to improve asthma and COPD care. ${ }^{24}$ Embedding the AR CDSS into the Allergy Diary Companion is expected to increase usage of the AR CDSS, resulting in improved standard of AR care in routine clinical practice, both at the physician and pharmacy levels. Indeed, CDSSs have already proved beneficial at the pharmacy level, ${ }^{25,26}$ reducing the frequency of drug-drug interactions and preventing inappropriate prescribing and underprescribing. ${ }^{27}$ It should also be noted that although a recently conducted Cochrane review provided no evidence that the use of electronic health information (EHI) translates into improved clinical practice or patient outcomes, it does suggest that when practitioners are provided with EHI and education or training, its use increases. ${ }^{28}$ It was further noted that for $\mathrm{EHI}$ to be applied in patient care, it will be necessary to understand why practitioners' are reluctant to apply EHI when treating people and to determine the most effective way(s) to reduce this reluctance. $^{28}$ 
(A)

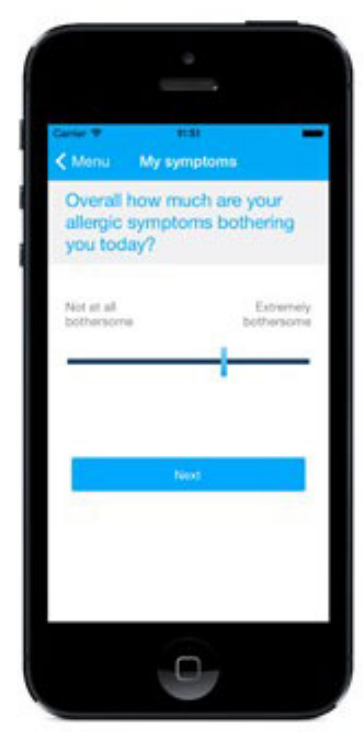

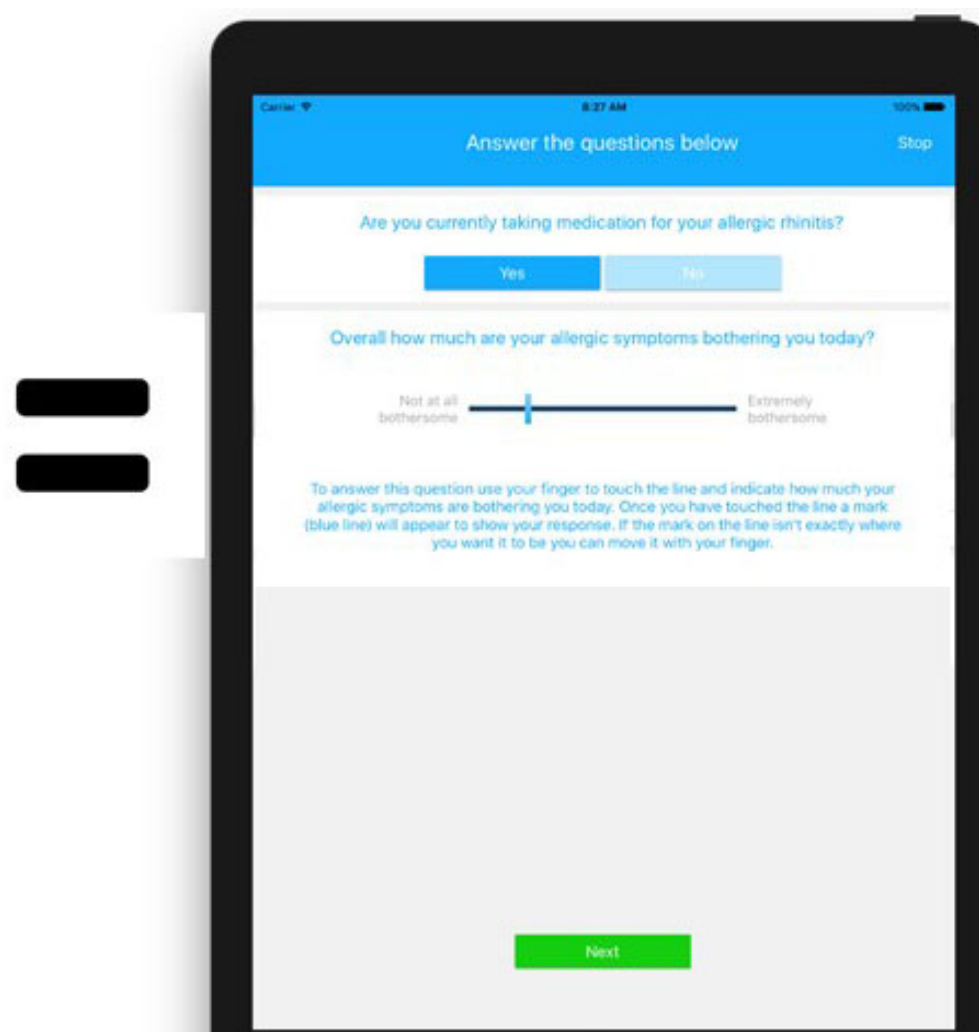

(B)
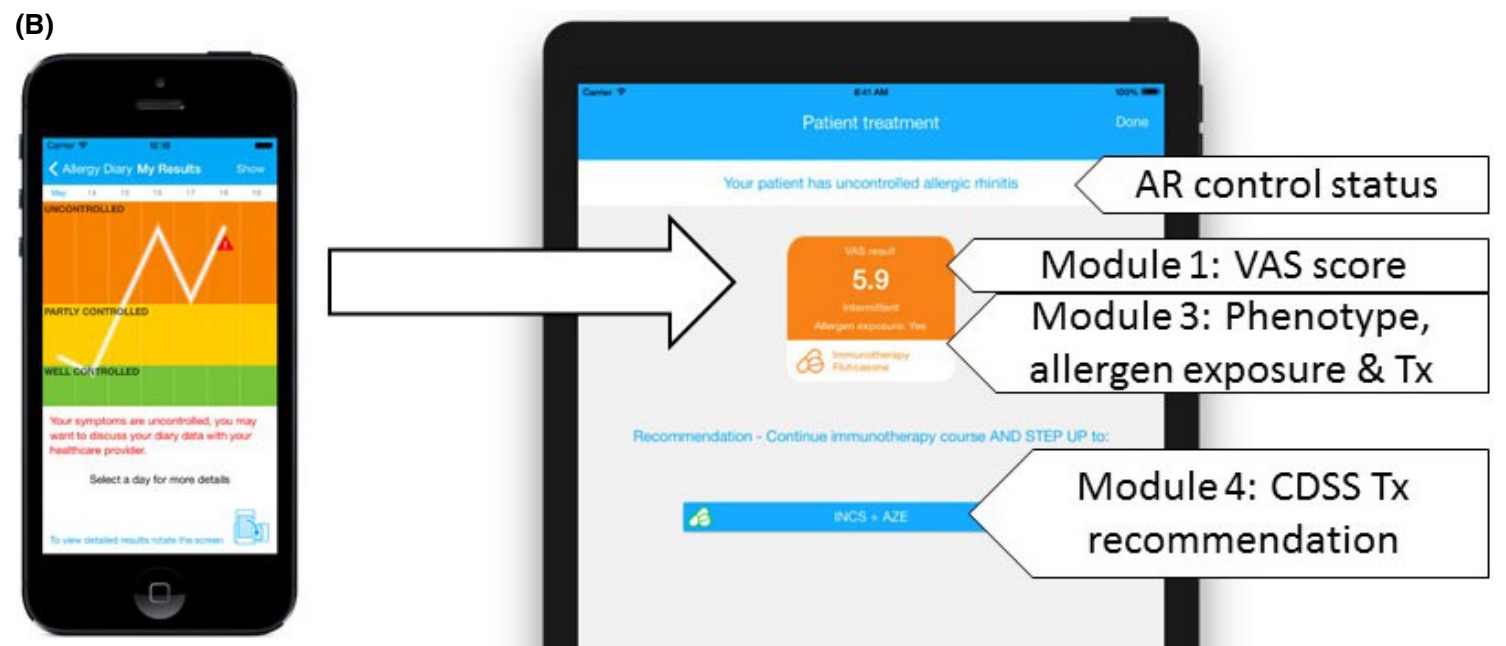

Module 4: CDSS Tx recommendation

FIGURE 3 (A) VAS interface of Allergy Diary and Allergy Diary Companion apps and (B) treatment recommendation provided by e-CDSS of the Allergy Diary Companion app, including information from Modules 1, 2 and 3 
On the patient side, mobile phone apps to improve allergy and asthma care are part of an ever-growing number of $\mathrm{m}$-tools available, but their usefulness is still debated and studies have been small. ${ }^{29,30}$ However, a large study including 327 individuals with AR or asthma, showed that QoL was improved in AR patients and the likelihood of asthma control increased when using an app which facilitated communication with physicians and which recorded health status and medication compliance. ${ }^{31}$

A limitation of the Allergy Diary Companion is that it currently relies on the input of information by patients themselves. It does not take into account data already recorded by patients in their Allergy Diary. In other words, there is a lack of connectivity between $\mathrm{m}$-health tools. One improvement could be to upload patient data directly from the Allergy Diary to the Allergy Diary Companion (ie Module 5; see Figure 4). This step would need serious ethical consideration that is currently being discussed. Personal data protection is a fundamental right in Europe, enshrined in Article 8 of the Charter of Fundamental Rights of the European Union, as well as in Article 16(1) of the Treaty on the Functioning of the European Union. Guidance already exists on data protection requirements for "apps" with Opinion 2/2013 of the Article 29 Working Party of 27 February 2013 on apps on smart devices. In the EU, the currently applicable Personal Data Protection Directive is being revised in order to better respond to challenges posed by the rapid development of new technologies and globalization while ensuring that individuals retain effective control over their personal data. The Allergy Diary Companion app will comply with all local and European directives. Future iterations of the Allergy Diary Companion may permit connectivity with pollen, air pollution, meteorologic storm warnings and Google Trends which may influence treatment recommendations. Finally, all Allergy Diary Companion app functionally and management advice needs to be validated, and this will be done prior to launch. The Allergy Diary Companion app is an ideal tool to assess the benefit of using the AR CDSS in a real-life setting. The value of this $m$-health tool is that it allows for variations and modifications of the e-CDSS based on real-life experience, thus moving from a consensus-based CDSS to an evidence-based one.

Other modules which may be used to "feed" the e-CDSS could include identification of those patients at risk of allergen exposure by incorporating a pollen alert module (Module 6; Figure 4) and a sentinel network module (Module 7; Figure 4). The sentinel network is an early warning system or predictor for patients of impending symptoms. A recently obtained $\mathrm{H} 2020$ grant (POLLAR: Impact of air pollution in asthma and rhinitis) will help to answer this question. This system should also prove useful in predicting asthma control deterioration in those with comorbid rhinitis. Use of predictive algorithms have already been used in home monitoring of asthma and chronic obstructive pulmonary disease patients, but with varying degrees of success, mainly due to poor patient compliance and poor performance of conventional algorithms for detecting deteriorations. ${ }^{32}$ These improvements to the system would essentially negate the need for Modules 1 and 3 for those users with smartphones. Modules 1 and 3 would remain in the system for those patients who do not have a smartphone or do not wish to download Allergy Diary. Finally, a PC-based tool should also be developed, enabling physicians to import data, with individual patient consent, to their pc directly from the patient's Allergy Diary, interacting with Allergy Diary Companion, so that data may be stored as an electronic file as part of the patient's notes.

When developing any new m-health tool, the aim is to achieve a high quality and popular app, which will be used enthusiastically and provide benefit to users. This is a challenging process, and not
FIGURE 4 Schematic representation of architecture of an improved e-CDSS. M, module; Tx, treatment; e-CDSS, electronic clinical decision support system
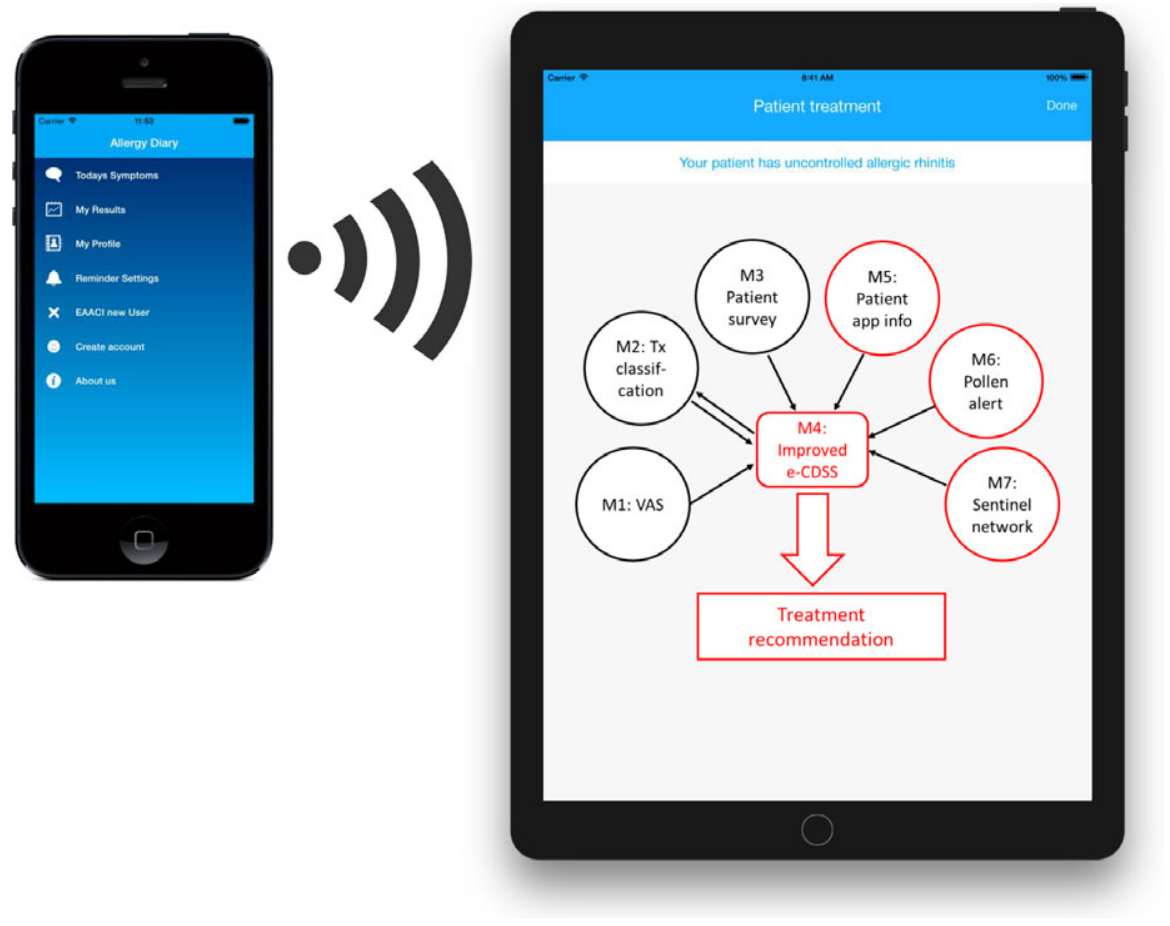
always entirely successful, either due to design issues ${ }^{33}$ or at the implementation stage. ${ }^{28,34}$ Allergy Diary Companion for providers has been designed to be used in conjunction with the Allergy Diary for patients as part of MASK (included in the B3 action plan of the European Innovation Partnership on Active and Healthy Ageing (EIA on AHA)). MASK follows the JA-CHRODIS (Joint Action on Chronic Diseases and Promoting Healthy Ageing across the Life Cycle) recommendations for the evaluation of good practices. This means that it works well, produces good results and is recommended as a model. ${ }^{35}$ MASK complies with equity (design and implementation), practice (eg appropriateness of design and SMART objectives), ethical, evaluation, empowerment/participation, target population, sustainability, governance and scalability targets. ${ }^{35}$ Use of both apps will (a) permit patients to screen for allergic disease and monitor AR and asthma control, (b) assist pharmacists in recommending over the counter medications and prompt referral of patients with uncontrolled AR to physicians and (c) encourage primary care physicians to prescribe appropriate treatment, to followup in accordance with the AR CDSS and to refer to specialist clinics when appropriate. ${ }^{36}$ However, the e-CDSS does not include information on allergic multimorbidities. ${ }^{37}$ That will be included at a later stage.

In conclusion, the Allergy Diary Companion for providers has been designed to provide healthcare information to practitioners and researchers, to permit real-time monitoring of disease-related data and to provide guideline and expert-endorsed AR treatment recommendations. The Allergy Diary Companion is currently being finalized and will be launched in 2018 .

\section{CONFLICT OF INTEREST}

The authors declare no conflict of interests.

\section{REFERENCES}

1. Global Initiative for Asthma. Global Strategy for Asthma Management and Prevention; 2018. http://ginasthma.org/2018-gina-reportglobal-strategy-for-asthma-management-and-prevention/. Accessed 18th April 2018.

2. Bousquet J, Schunemann $H$, Arnavielhe $S$, et al. MACVIA clinical decision algorithm in allergic rhinitis in adolescents and adults. $J$ Allergy Clin Immunol. 2016;138:367-374.

3. Hellings PW, Fokkens WJ, Akdis C, et al. Uncontrolled allergic rhinitis and chronic rhinosinusitis: where do we stand today? Allergy. 2013;68:1-7.

4. Hellings PW, Muraro A, Fokkens W, et al. A common language to assess allergic rhinitis control: results from a survey conducted during EAACl 2013 Congress. Clin Transl Allergy. 2015;5:36.

5. Bousquet J, Bachert $C$, Price $D$, et al. Assessing allergic rhinitis symptom control using a simple visual analogue scale: the digital solution. Allergy 2014;69:A277.

6. Bousquet J, Schunemann HJ, Fonseca J, et al. MACVIA-ARIA Sentinel NetworK for allergic rhinitis (MASK-rhinitis): the new generation guideline implementation. Allergy. 2015;70:1372-1392.

7. Bousquet J, Bewick M, Arnavielhe $\mathrm{S}$, et al. Work productivity in rhinitis using cell phones: the MASK pilot study. Allergy. 2017;72: 1475-1484.
8. Bousquet J, Caimmi DP, Bedbrook A, et al. Pilot study of mobile phone technology in allergic rhinitis in European countries: the MASK-rhinitis study. Allergy. 2017;72:857-865.

9. De Greve G, Hellings PW, Fokkens WJ, Pugin B, Steelant B, Seys SF. Endotype-driven treatment in chronic upper airway diseases. Clin Transl Allergy. 2017;7:22.

10. Hellings PW, Borrelli D, Pietikainen S, et al. European summit on the prevention and self-management of chronic respiratory diseases: report of the european union parliament summit (29 March 2017) Clin Transl Allergy. 2017;7:49.

11. Muraro A, Roberts $G$, Halken $S$, et al. EAACl guidelines on allergen immunotherapy: executive statement. Allergy 2018;73:739-743. https://doi.org/10.111/all.13420 [ePub ahead of print]

12. Bousquet PJ, Demoly P, Devillier P, Mesbah K, Bousquet J. Impact of allergic rhinitis symptoms on quality of life in primary care. Int Arch Allergy Immunol. 2013;160:393-400.

13. Bousquet PJ, Bachert C, Canonica GW, et al. Uncontrolled allergic rhinitis during treatment and its impact on quality of life: a cluster randomized trial. J Allergy Clin Immunol. 2010;126(666-8):e1-e5.

14. Caimmi D, Baiz N, Tanno LK, et al. Validation of the MASK-rhinitis visual analogue scale on smartphone screens to assess allergic rhinitis control. Clin Exp Allergy. 2017;47:1526-1533.

15. Demoly P, Bousquet PJ, Mesbah K, Bousquet J, Devillier P. Visual analogue scale in patients treated for allergic rhinitis: an observational prospective study in primary care: asthma and rhinitis. Clin Exp Allergy. 2013;43:881-888.

16. Fonseca JA, Nogueira-Silva L, Morais-Almeida M, et al. Validation of a questionnaire (CARAT10) to assess rhinitis and asthma in patients with asthma. Allergy. 2010;65:1042-1048.

17. Rouve S, Didier A, Demoly P, Jankowsky R, Klossek JM, AnnesiMaesano I. Numeric score and visual analog scale in assessing seasonal allergic rhinitis severity. Rhinology. 2010;48:285-291.

18. Tricco AC, Ashoor HM, Cardoso R, et al. Sustainability of knowledge translation interventions in healthcare decision-making: a scoping review. Implement Sci. 2016;11:55.

19. Stacey D, Hawker G, Dervin G, et al. Decision aid for patients considering total knee arthroplasty with preference report for surgeons: a pilot randomized controlled trial. BMC Musculoskelet Disord. 2014;15:54

20. van Weert JC, van Munster BC, Sanders R, Spijker R, Hooft L, Jansen J. Decision aids to help older people make health decisions: a systematic review and meta-analysis. BMC Med Inform Decis Mak 2016;16:45

21. Baiardini I, Braido F, Bonini M, Compalati E, Canonica GW. Why do doctors and patients not follow guidelines? Curr Opin Allergy Clin Immunol. 2009;9:228-233.

22. Mickan S, Atherton H, Roberts NW, Heneghan C, Tilson JK. Use of handheld computers in clinical practice: a systematic review. BMC Med Inform Decis Mak. 2014;14:56.

23. Matui P, Wyatt JC, Pinnock H, Sheikh A, McLean S. Computer decision support systems for asthma: a systematic review. NPJ Prim Care Respir Med. 2014;24:14005.

24. Fathima M, Peiris D, Naik-Panvelkar P, Saini B, Armour CL. Effectiveness of computerized clinical decision support systems for asthma and chronic obstructive pulmonary disease in primary care: a systematic review. BMC Pulm Med. 2014;14:189.

25. Watkins K, Wood H, Schneider CR, Clifford R. Effectiveness of implementation strategies for clinical guidelines to community pharmacy: a systematic review. Implement Sci. 2015;10:151.

26. Bertsche T, Nachbar M, Fiederling J, et al. Assessment of a computerised decision support system for allergic rhino-conjunctivitis counselling in German pharmacy. Int J Clin Pharm. 2012;34:17-22.

27. Curtain C, Peterson GM. Review of computerized clinical decision support in community pharmacy. J Clin Pharm Ther. 2014;39:343348. 
28. Fiander M, McGowan J, Grad R, et al. Interventions to increase the use of electronic health information by healthcare practitioners to improve clinical practice and patient outcomes. Cochrane Database Syst Rev. 2015;14:CD004749.

29. Huang X, Matricardi PM. Allergy and asthma care in the mobile phone era. Clin Rev Allergy Immunol. 2016; https://doi.org/10.1007/ s12016-016-8542-y [last accessed 02.08.18]

30. Miller L, Schuz B, Walters J, Walters EH. Mobile technology interventions for asthma self-management: systematic review and metaanalysis. JMIR Mhealth Uhealth. 2017;5:e57.

31. Cingi C, Yorgancioglu A, Cingi CC, et al. The "physician on call patient engagement trial" (POPET): measuring the impact of a mobile patient engagement application on health outcomes and quality of life in allergic rhinitis and asthma patients. Int Forum Allergy Rhinol. 2015;5:487-497.

32. Sanchez-Morillo D, Fernandez-Granero MA, Leon-Jimenez A. Use of predictive algorithms in-home monitoring of chronic obstructive pulmonary disease and asthma: A systematic review. Chron Respir Dis. 2016;13:264-283.

33. Burnay E, Cruz-Correia R, Jacinto T, Sousa AS, Fonseca J. Challenges of a mobile application for asthma and allergic rhinitis patient enablement-interface and synchronization. Telemed J E Health. 2013;19:13-18.

34. Ross J, Stevenson F, Lau R, Murray E. Factors that influence the implementation of e-health: a systematic review of systematic reviews (an update). Implement Sci. 2016;11:146.
35. Bousquet J, Onorato GL, Bachert C, et al. CHRODIS criteria applied to the MASK (MACVIA-ARIA Sentinel NetworK) Good Practice in allergic rhinitis: a SUNFRAIL report. Clin Transl Allergy. 2017;7:37.

36. Bourret R, Bousquet J, Mercier J, et al. MASK-rhinitis, a single tool for integrated care pathways in allergic rhinitis. World Hosp Health Serv. 2015;51:6-9.

37. Cingi C, Gevaert P, Mosges R, et al. Multi-morbidities of allergic rhinitis in adults: European Academy of Allergy and Clinical Immunology Task Force Report. Clin Transl Allergy. 2017;7:17.

\section{SUPPORTING INFORMATION}

Additional supporting information may be found online in the Supporting Information section at the end of the article.

How to cite this article: Courbis A-L, Murray R, Arnavielhe S, et al. Electronic clinical decision support system for allergic rhinitis management. Clin Exp Allergy. 2018;48:1640-1653.

https://doi.org/10.1111/cea.13230 